\title{
Pre-existing antibodies against polyethylene glycol reduce asparaginase activities on first administration of pegylated $E$. coli asparaginase in children with acute lymphocytic leukemia
}

\begin{abstract}
Alaeddin Khalil, ${ }^{1}$ Gudrun Würthwein, ${ }^{1}$ Jana Golitsch, ${ }^{1}$ Georg Hempel, ${ }^{2}$ Manfred Fobker, ${ }^{3}$ Joachim Gerss, ${ }^{4}$ Anja Möricke, ${ }^{5}$ Martin Zimmermann, ${ }^{6}$ Petr Smisek, ${ }^{7}$ Massimo Zucchetti, ${ }^{8}$ Christa Nath, ${ }^{9}$ Andishe Attarbaschi, ${ }^{10}$ Arend von Stackelberg, ${ }^{11}$ Nicola Gökbuget, ${ }^{12}$ Carmelo Rizzari, ${ }^{13}$ Valentino Conter, ${ }^{13}$ Martin Schrappe, ${ }^{5}$ Joachim Boos ${ }^{1}$ and Claudia Lanvers-Kaminsky ${ }^{1}$

${ }^{1}$ Department of Pediatric Hematology and Oncology, University Children's Hospital Münster, Münster, Germany; ${ }^{2}$ Department of Pharmaceutical and Medical Chemistry, Clinical Pharmacy, University of Münster, Münster, Germany; ${ }^{3}$ Center of Laboratory Medicine, University Hospital Münster, Albert-Schweitzer-Campus 1, Münster, Germany; ${ }^{4}$ Institute of Biostatistics and Clinical Research, University of Münster, Münster, Germany; ${ }^{5}$ Department of Pediatrics, University Medical Center SchleswigHolstein, Campus Kiel, Kiel, Germany: ${ }^{6}$ Department of Pediatric Hematology and Oncology, Medical School Hannover, Hannover, Germany; ${ }^{7}$ Department of Pediatric Hematology and Oncology, University Hospital Motol, Praha, Czech Republic; ${ }^{8}$ Laboratory of Cancer Pharmacology, Department of Oncology, Istituto di Ricerche Farmacologiche Mario Negri IRCCS, Milan, Italy; ${ }^{9}$ Departments of Biochemistry and Oncology, The Children's Hospital at Westmead, Sydney Pharmacy School, University of Sydney, Sydney, Australia; ${ }^{10}$ Department of Pediatric Hematology and Oncology, St. Anna Children's Hospital, Department of Pediatrics and Adolescent Medicine, Medical University of Vienna, Vienna, Austria; ${ }^{11}$ Departments of Pediatric Oncology/Hematology and of General Pediatrics, Charité -University Medicine Berlin, Berlin, Germany; ${ }^{12}$ Department of Medicine, University Hospital, Frankfurt am Main, Germany and ${ }^{13}$ Pediatric Hematology-Oncology Unit, Department of Pediatrics, University of MilanoBicocca, MBBM Foundation, ASST-Monza, Monza, Italy
\end{abstract}

\section{ABSTRACT}

A ntibodies against polyethylene glycol (PEG) in healthy subjects raise concerns about the efficacy of pegylated drugs. We evaluated the prevalence of antibodies against PEG among patients with acute lymphoblastic leukemia (ALL) prior to and/or immediately after their first dose of pegylated E.coli asparaginase (PEG-ASNase). Serum samples from 701 children (673 with primary ALL, 28 with relapsed ALL) and 188 adults with primary ALL were analyzed for anti-PEG IgG and IgM. Measurements in 58 healthy infants served as a reference to define cut-points for antibody-positive and -negative samples. The prevalence of anti-PEG antibodies in ALL patients prior to the first administration of PEG-ASNase was $13.9 \%$ for anti-PEG IgG and $29.1 \%$ for anti-PEG IgM. After administration of PEG-ASNase the prevalence of anti-PEG antibodies decreased to $4.2 \%$ for anti-PEG IgG and to $4.5 \%$ for anti-PEG IgM. Pre-existing anti-PEG antibodies did not inhibit PEGASNase activity but significantly reduced PEG-ASNase activity levels in a concentration-dependent manner. Although pre-existing anti-PEG antibodies were not boosted, pre-existing anti-PEG IgG were significantly associated with first-exposure hypersensitivity reactions (Common Terminology Criteria for Adverse Events grade 2) $(P<0.01$; Fisher exact test). Two of four patients with pre-existing anti-PEG IgG and first-exposure hypersensitivity reactions were not switched to Erwinia ASNase and continued on PEG-ASNase with sufficient activity $(\geq 100 \mathrm{U} / \mathrm{L})$. In conclusion, pre-existing anti-PEG antibodies were detected in a considerable proportion of patients with ALL and although they did not inhibit PEG-ASNase activity, they were associated with lower
Haematologica 2022

Volume 107(1):49-57

\section{Correspondence:}

CLAUDIA LANVERS-KAMINSKY

lanvers@uni-muenster.de

Received: May 19, 2020.

Accepted: November 25, 2020.

Pre-published: December 10, 2020

https://doi.org/10.3324/haematol.2020.258525

(C)2022 Ferrata Storti Foundation

Material published in Haematologica is covered by copyright. All rights are reserved to the Ferrata Storti Foundation. Use of published material is allowed under the following terms and conditions:

https://creativecommons.org/licenses/by-nc/4.0/legalcode. Copies of published material are allowed for personal or internal use. Sharing published material for non-commercial purposes is subject to the following conditions:

https://creativecommons.org/licenses/by-nc/4.0/legalcode, sect. 3. Reproducing and sharing published material for commercial purposes is not allowed without permission in writing from the publisher. 
serum PEG-ASNase activity levels. Patients with pre-existing antibodies may show mild to moderate signs of hypersensitivity reaction after their first administration PEG-ASNase, which may be successfully addressed by re-challenge.

\section{Introduction}

Due to its favorable toxicity profile polyethylene glycol (PEG) is widely used in foods, cosmetics, and pharmaceuticals. ${ }^{1}$ Pegylation can improve the therapeutic benefit of protein drugs. It prolongs their elimination by increasing the molecular mass and protecting them from enzymatic cleavage and it decreases their immunogenicity by shielding potential antigenic epitopes. ${ }^{2-4}$ Numerous pegylated drugs are currently marketed in the USA and Europe including pegylated uricase (Krystexxa ${ }^{\mathrm{TM}}$ ), pegylated interferon (Pegasys $^{\mathrm{TM}}$, PegIntron ${ }^{\mathrm{TM}}$ ) and pegylated E. coli asparaginase (PEG-ASNase) (Oncaspar ${ }^{\mathrm{TM}}$, calaspargase, Asparlas $\left.^{\mathrm{TM}}\right)^{5.8}$ The asparagine-hydrolyzing enzyme asparaginase (ASNase) is crucial for the successful treatment of acute lymphoblastic leukemia (ALL), 10 and because of its favorable drug characteristics PEG-ASNase is increasingly replacing its unmodified native form in frontline treatment of ALL. ${ }^{11-13}$

While pegylated proteins, despite their higher molecular mass, tend to be less immunogenic than their non-pegylated forms of protein drugs, antibodies against PEG have been detected in patients treated with pegylated proteins as well as in healthy volunteers. ${ }^{14}$ The reported prevalence varies widely between studies $(0.2-72 \%)$ which is partly due to the use of different detection methods and cutpoint definitions (Online Supplementary Tables S1 and S2). In animal studies anti-PEG antibodies, especially anti-PEG IgM, were considered responsible for the accelerated blood clearance of pegylated proteins, liposomes, and nanoparticles. ${ }^{15,16}$ In human studies, the reported effects of anti-PEG antibodies on the therapeutic efficacy of pegylated drugs have been ambiguous; no effects of antibodies against PEG have been observed for pegylated interferons to date, ${ }^{17}$ whereas in patients with gout anti-PEG IgM and anti-PEG IgG were associated with a faster elimination of PEG-uricase. ${ }^{18,19}$ Drug authorities now require evaluation of the relevance of anti-PEG antibodies during drug development and registration processes. ${ }^{20,21}$

Published data suggest that anti-PEG antibodies may have important effects on the efficacy of PEG-ASNase. Armstrong et al. detected anti-PEG antibodies in 12 of 15 patients with undetectable ASNase activities after PEGASNase administration and also in four of 12 patients before their first PEG-ASNase administration. ${ }^{22}$ Liu et al. recently showed that anti-PEG ASNase antibodies consisted mainly of antibodies against PEG rather than E. coli

Table 1. Demographics of the patients in the three cohorts of acute lymphocytic leukemia cases.

\begin{tabular}{|c|c|c|c|}
\hline Protocol & $\begin{array}{c}\text { ALL-cohort } 1 \\
\text { AIEOP-BFM ALL } 2009\end{array}$ & $\begin{array}{c}\text { ALL-cohort } 2 \\
\text { ALL-REZ BFM } 2002 \& \\
\text { ALL-REZ BFM Observ. }\end{array}$ & $\begin{array}{l}\text { ALL-cohort } 3 \\
\text { GMALL 07/2003 }\end{array}$ \\
\hline Number & 673 & 28 & 188 \\
\hline $\operatorname{Sex}(M / F)$ & $401 / 272$ & $19 / 9$ & $120 / 68$ \\
\hline $\begin{array}{c}\text { Age, years } \\
\text { Median } \\
\text { Range }\end{array}$ & $\begin{array}{c}5.6 \\
1-18\end{array}$ & $\begin{array}{c}8.5 \\
5-17\end{array}$ & $\begin{array}{c}36 \\
18-74\end{array}$ \\
\hline
\end{tabular}

abservational Study and Biobank. M: male; F: female.
ASNase and were significantly associated with hypersensitivity reactions to PEG ASNase. ${ }^{23}$

Given the increasing use of PEG-ASNase in frontline treatment for ALL, the aims of this study were to: (i) evaluate the prevalence of anti-PEG antibodies in three cohorts of patients (children and adults with primary ALL and children with relapsed ALL) before and/or immediately after their first dose of PEG-ASNase during induction treatment, and (ii) investigate the effects of pre-existing anti-PEG antibodies on PEG-ASNase activities and hypersensitivity reactions.

\section{Methods}

\section{Patients}

Samples for anti-PEG antibody determination were obtained from children with primary ALL (ALL-cohort 1), children with relapsed ALL (ALL-cohort 2), adults with primary ALL (ALLcohort 3 ) and healthy infants, who served as the reference cohort. Patients in ALL-cohort 1 were treated according to the AIEOPBFM ALL 2009 trial (ClinicalTrials.gov identifier: NCT01117441) and a total of 673 plasma samples were collected from 673 pediatric patients (401 males, 272 females) prior to their first administration of PEG-ASNase. In addition, 646 patients provided one or two more serum samples (1,183 in total) taken within 15 days after the first PEG-ASNase dose on day 12 of induction.

Patients in ALL-cohort 2 were diagnosed with relapsed ALL and treated according to the protocol of the ALL-REZ BFM 2002 (ClinicalTrials.gov identifier: 00114348) or the ALL-REZ BFM Observational Study and Biobank study. Twenty-eight samples were collected from 28 patients ( 19 males, 9 females) 0 to 2 days after the first dose of PEG-ASNase

Patients in ALL-cohort 3 were treated according to the multicenter GMALL 07/2003 trial (ClinicalTrials.gov identifier: 00198991). A total of 188 samples from 120 males and 68 females were taken on the same day after the first administration of PEGASNase $(n=16)$ or the following day $(n=172)$. Further details on the ALL cohorts are provided in Table 1 and in the Online Supplementary Data.

The respective ALL studies were approved by national and local review boards in accordance with the Helsinki Declaration and national laws. The approvals included monitoring antibodies against PEG-ASNase and determination of ASNase activity. Patients and/or their guardians gave their signed informed consent to participate in the monitoring of ASNase activities and antibodies against PEG-ASNase.

Serum samples from 58 infants $<1$ year, who were considered naive to PEG were used as reference. The Central Laboratory of the University Hospital Muenster provided anonymized remainders of routine serum samples from infants. Only age in months was disclosed. Thus, these samples were considered as completely anonymized leftover material.

\section{Determination of antibodies against PEG}

For the detection of anti-PEG IgG and anti-PEG IgM the flow cytometry method described by Armstrong et al..$^{22}$ was transferred to a 96-well format with fluorescent read-out. TentaGel M OCH. particles $(10 \mu \mathrm{m})$, to which methoxy-polyethylene glycol chains with a mean molecular weight of 5,000 Da were covalently 
bound, were used as the antigen (RAPP Polymere, Tuebingen, Germany). Mean fluorescent intensities (MFI) of duplicate determinations were calculated for anti-PEG IgG and IgM levels. Based on the MFI, determined in the reference cohort, cut-points of 8 (anti-PEG IgG) and 2 (anti-PEG IgM) were defined to classify samples as positive or negative. A detailed description of the method for the determination of anti-PEG antibodies and its performance characteristics is included in the Online Supplementary Data along with a description of the measurement of PEG-ASNase activity and total $\operatorname{IgG}$ and $\operatorname{IgM}$.

\section{Statistics}

Statistical analyses were performed using SAS $^{\circledR}$ Version 9.4 (SAS Institute Inc., Cary, NC, USA) and RStudio Version 1.2.5033 (RStudio: Integrated Development for R. RStudio, Inc., Boston, MA, USA. URL: http://www.rstudio.com/). Kruskal-Wallis one way analysis of variance on ranks, all pairwise multiple comparison procedures (Dunn method, Holm-Sidak method), the MannWhitney rank sum test, Wilcoxon signed-rank test, $\chi^{2}$ test, McNemar $\chi^{2}$ test with continuity correction, Fisher exact test, Pearson correlation and logistic regression were used as indicated.

\section{Results}

\section{Anti-PEG IgG and anti-PEG IgM antibody levels}

The MFI for anti-PEG IgG was between 0.65 and 67.4, whereas that for anti-PEG IgM was between 0.13 and 30.8 . Overall, anti-PEG IgG levels correlated with anti-PEG IgM levels ( $\mathrm{r}=0.68, P<0.005$, Pearson correlation). However, high anti-PEG IgG levels did not necessarily coincide with high anti-PEG IgM levels and vice versa (Online Supplementary Tables S3 and S4). On average the lowest levels of anti-PEG IgG and IgM were determined in the reference cohort and the highest levels in children with primary ALL prior to their first dose of PEG-ASNase (ALLcohort 1) (Figure 1).

In ALL-cohort 1 anti-PEG IgG and IgM levels were significantly lower after the administration of PEG-ASNase. This difference was statistically significant in an unpaired analysis, when all samples available after the first administration were included $(n=1,183$, median days after administration: 13 ; range: $1-15 ; P<0.001$, Mann-Whitney rank sum test) and in a paired analysis, when only the first sample taken after administration was chosen for the pairwise comparison ( $\mathrm{n}=646$, median days after administration: 7; range: $1-15 ; P<0.001$, Wilcoxon signed-rank test). In addition, anti-PEG IgG and IgM levels were also significantly lower in ALL-cohorts 2 and 3, which were analyzed after PEG-ASNase administration $(P<0.001$, Kruskal-Wallis oneway analysis of variance on ranks, all pairwise multiple comparison procedures [Dunn method]) (Figure 1). The prevalence of anti-PEG antibodies was correspondingly lower in samples/patients analyzed after administration of PEG-ASNase (Figure 2C-E). In ALL-cohort $113.9 \%$ of samples were positive for anti-PEG IgG and $29.1 \%$ positive for anti-PEG IgM prior to the administration of PEG-ASNase. After administration of PEG-ASNase the prevalence dropped to $4.2 \%$ for anti-PEG IgG and $4.5 \%$ for anti-PEG IgM. This represented a significant reduction in prevalence by PEG-ASNase administration $\left(P<0.0001, \mathrm{McNemar} \chi^{2}\right.$ test with continuity correction) (Figure $2 \mathrm{~B}, \mathrm{C})$. Among
A

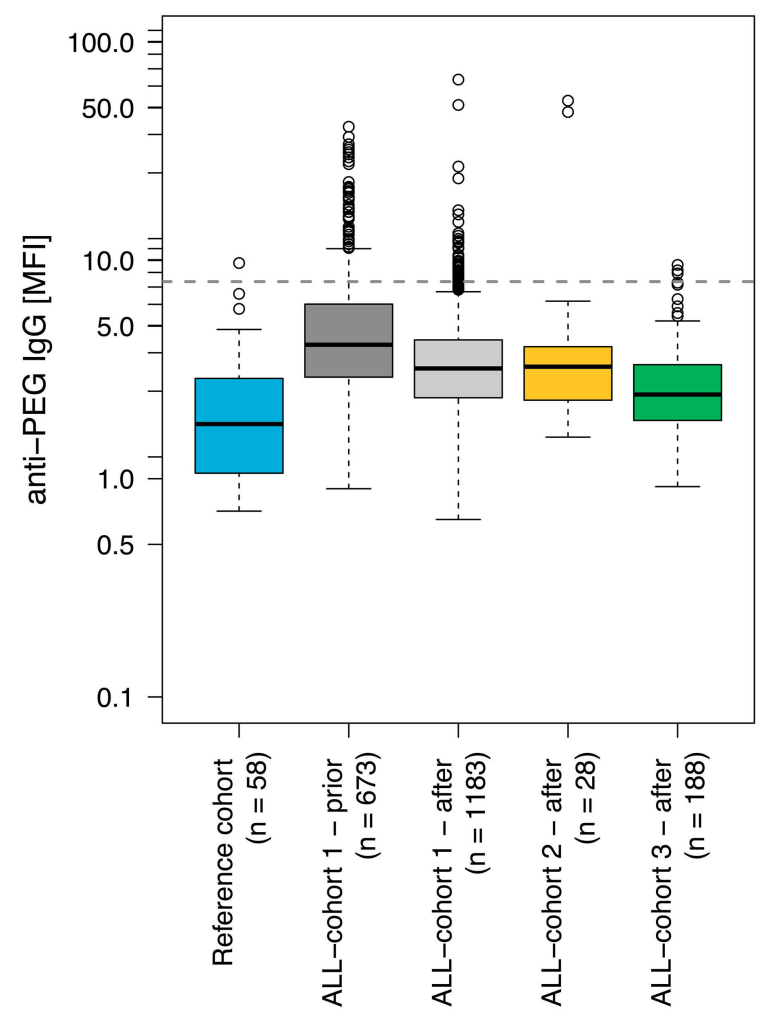

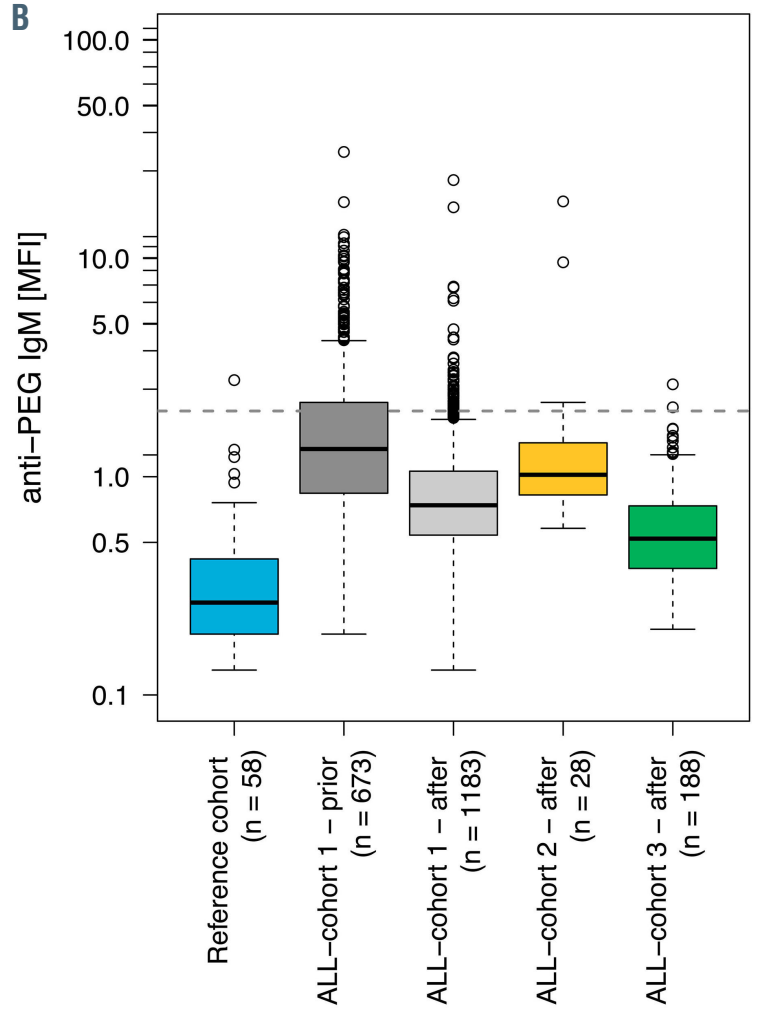

Figure 1. Box plots of anti-PEG antibodies in the reference and acute lymphocytic leukemia cohorts. (A, B) Box plots of anti-PEG IgG (A) and anti-PEG IgM (B) mean fluorescent intensities (MFI) determined in the reference and the acute lymphocytic leukemia (ALL) cohorts. The boxes represent the first and third quartiles, the lines in the box the represent the medians, the whiskers the first quartile - (1.5 $\mathrm{x}$ the interquartile range between the first and third quartiles [IQR]) and the third quartile $+(1.5 \times \mathrm{IQR})$, and the dots the outliers. The dashed reference lines represent the cut-points for anti-PEG IgG $(\mathrm{MFI}=8)(\mathrm{A})$ and anti-PEG IgM $(\mathrm{MFI}=2)(\mathrm{B})$. 
A

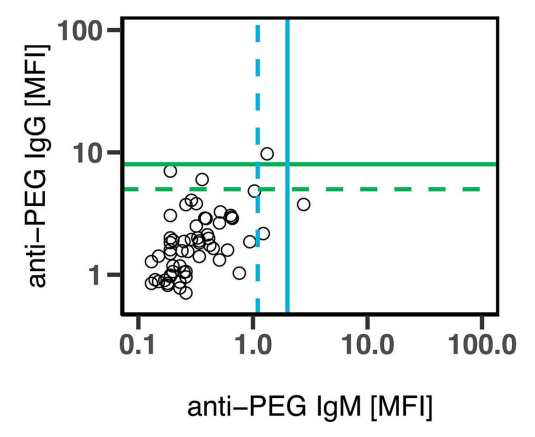

B

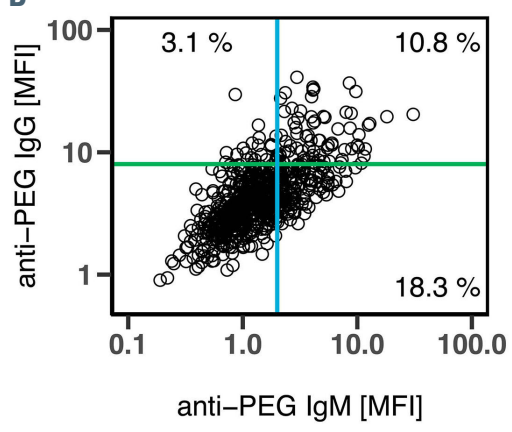

D

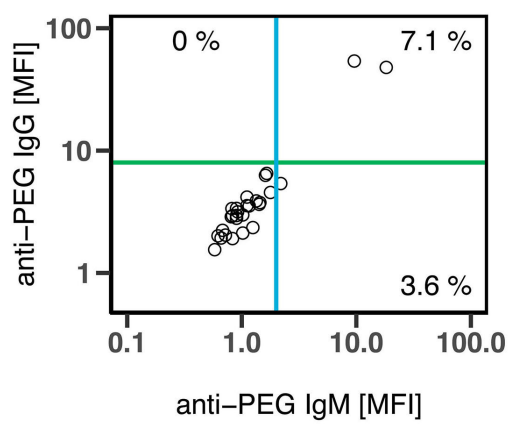

C

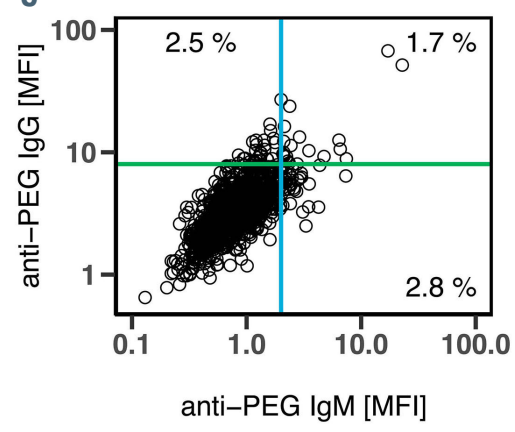

$\mathrm{E}$

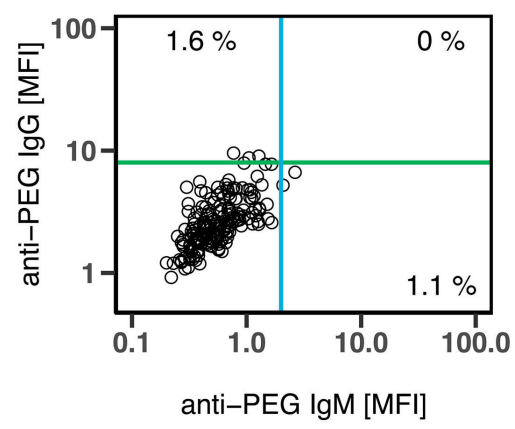

Figure 2. Scatter plots of anti-PEG IgM versus anti-PEG IgG levels determined in the reference and the acute lymphocytic leukemia cohorts. (A) Samples of the reference cohort were taken from healthy infants. (B, C) Samples of acute lymphocytic leukemia (ALL)-cohort 1 were taken from patients treated according to the AIEOPBFM ALL 2009 trial and were either collected prior to their first dose of PEG-asparatinase (ASNase) (B) or within 15 days after administration of the first PEG-ASNase dose (C). (D, E) Samples from ALL-cohort 2 (children with relapsed ALL treated according to the ALL-REZ BFM 2002 and the ALL-REZ BFM ALL observational study and biobank) (D) and samples from ALL-cohort 3 (adults treated according to the GMALL 07/2003 protocol) (E) were taken after administration of PEG-ASNase. AntiPEG antibodies were determined by the level of mean fluorescent intensity (MFI). In (A) the dashed light blue vertical line represents the $95^{\text {th }}$ percentile of anti-PEG IgM MFI and the dashed green horizontal line represents the $95^{\text {th }}$ percentile of anti-PEG IgG MFI determined in the reference cohort. The solid reference lines represent the defined cut-points after visual adjustment for anti-PEG IgM (light blue vertical line, MFI $=2$ ) and anti-PEG IgG (green horizontal line, MFI = 8).

patients with pre-existing anti-PEG antibodies, the antibody levels decreased a mean of about 2.7-fold for antiPEG IgG and about 4.1-fold for anti-PEG IgM.

\section{PEG-asparaginase activities}

PEG-ASNase activities were determined in 1,183 samples from 646 patients of ALL-cohort 1 . Samples were collected within 15 days of the first administration of 2,500 $\mathrm{U} / \mathrm{m}^{2} \mathrm{PEG}$-ASNase (maximum 3,750 U per dose) on day 12 of induction. Of these samples, $95.5 \%$ were collected at the scheduled times (day $7 \pm 1$ and day $14 \pm 1$ after administration). The mean ( \pm standard deviation) PEG-ASNase activities determined were $911 \pm 311 \mathrm{U} / \mathrm{L}$ on day $7 \pm 1$ and $527 \pm 200 \mathrm{U} / \mathrm{L}$ on day $14 \pm 1$.

PEG-ASNase activities were significantly lower among patients with elevated anti-PEG IgG $(\mathrm{MFI} \geq 8)$ or anti-PEG $\operatorname{IgM}(\mathrm{MFI} \geq 2)$ prior to their first dose of PEG-ASNase $(P<0.05$, Kruskal-Wallis one way analysis of variance on ranks, all pairwise multiple comparison procedures [HolmSidak method]). In addition, the PEG-ASNase activities decreased with increasing anti-PEG antibody levels prior to administration (Figure 3). To evaluate the effect of antiPEG antibodies on PEG-ASNase activities in individual patients, mean PEG-ASNase activities were calculated for the respective day after administration and individual PEGASNase activities were categorized as above or below the respective means. Pre-existing anti-PEG IgG (MFI $\geq 8)$ as well as pre-existing anti-PEG IgM (MFI $\geq 2)$ increased the risk of PEG-ASNase activities below average (anti-PEG
IgG: odds ratio [OR]: 2.06, 95\% confidence interval [95\% CI]: 1.44-2.96; anti-PEG IgM: OR: 1.65; 95\% CI: 1.27-2.15; $P<0.001, \chi^{2}$ test). No such associations were observed for anti-PEG IgG and IgM levels determined after PEG-ASNase administration. Pre-existing anti-PEG antibodies reduced but did not eliminate PEG-ASNase activities (Figure 3). Comparing the distribution of PEG-ASNase activities above and below $400 \mathrm{U} / \mathrm{L}, 100 \mathrm{U} / \mathrm{L}$ and the lower limit of quantitation (LLOQ $=5 \mathrm{U} / \mathrm{L})$, significantly more samples with PEG-ASNase activities <400 U/L were found in patients with already existing anti-PEG antibodies (Table 2). No differences were observed for the distribution of PEG-ASNase activities above and below $100 \mathrm{U} / \mathrm{L}$ and the LLOO (Table 2). Thus, silent inactivation of PEG-ASNase, which is defined by PEG-ASNase activities $<100 \mathrm{U} / \mathrm{L}$ within $7 \pm 1$ days and/or undetectable PEG-ASNase activities within $14 \pm 1$ days after administration without signs of hypersensitivity reaction, was not affected by pre-existing anti-PEG antibodies. ${ }^{24,25}$ Anti-PEG antibodies did not inhibit the catalytic activity of PEG-ASNase and anti-PEG IgG and/or IgM had no effect on asparagine hydrolysis by PEGASNase (Online Supplementary Figure S4).

\section{Anti-PEG antibodies prior to treatment with PEG-asparaginasese and hypersensitivity reactions to PEG-asparaginase}

After initial exposure to PEG-ASNase, seven patients in ALL-cohort $1(1.0 \%)$ showed hypersensitivity reactions (all Common Terminology Criteria for Adverse Events 

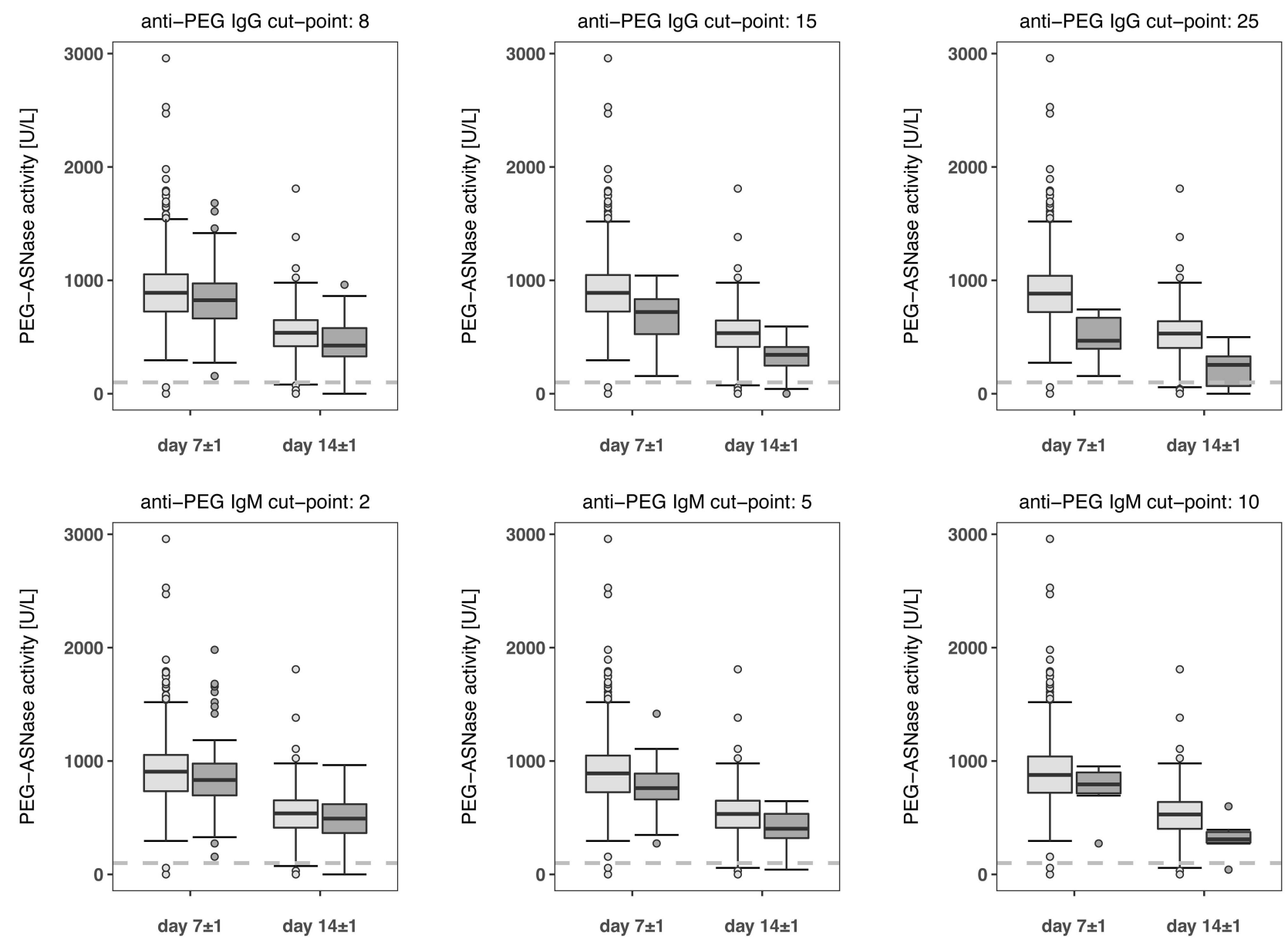

Figure 3. Box plots of PEG-asparaginase activities after the first dose of the drug. PEG-asparaginase (ASNase) activities were determined in patients of acute lymphoblastic leukemia (ALL) cohort 1 on day $7 \pm 1$ and day $14 \pm 1$ after administration of the first dose of PEG-ASNase and grouped according to various cut-points for pre-existing anti-PEG IgG and IgM mean fluorescent intensity (MFI). The light gray boxes represent PEG-ASNase activities determined in patients with anti-PEG antibody levels below the respective cut-point and the dark gray boxes represent PEG-ASNase activities determined in patients with anti-PEG antibody levels above the respective cut-point. The boxes represent the first and third quartiles, the lines in the box the medians, the whiskers the first quartile - (1.5 $x$ the interquartile range between the first and third quartiles [IQR]) and the third quartile + (1.5 x IQR), and the dots the outliers. The dashed reference line represents the target PEG-ASNase activity of $100 \mathrm{U} / \mathrm{L}$.

[CTCAE] grade 2) which were significantly associated with pre-existing anti-PEG IgG levels. Four of seven patients had pre-existing anti-PEG IgG $(M F I \geq 8)$ before their first PEG-ASNase dose (Table 3). No pre-existing antiE. coli ASNase antibodies were detected in these patients. Four patients ( 2 with and 2 without pre-existing anti-PEG antibodies) were switched to Erwinia ASNase. Among the four patients with a first-exposure hypersensitivity reaction and pre-existing anti-PEG IgG no further boosts of anti-PEG IgG levels were observed. The two patients with pre-existing anti-PEG IgG, who continued on PEGASNase, completed the scheduled PEG-ASNase treatment without further signs of hypersensitivity. The relative risk of a hypersensitivity reaction upon first exposure to PEGASNase was 8 times higher for patients with anti-PEG IgG $\mathrm{MFI} \geq 8$ and 50 times higher for patients with anti-PEG IgG MFI $\geq 25$ prior to their first PEG-ASNase (Table 3 ). This association was only observed for pre-existing anti-PEG IgG and not for pre-existing anti-PEG IgM.

\section{Discussion}

We detected a high prevalence of anti-PEG IgG (13.9\%) and $\operatorname{IgM}(29.1 \%)$ among children with primary ALL prior to their first PEG-ASNase.

Antibodies against PEG had already been detected in healthy volunteers of different ages and ethnicity and in patients who had never been treated with pegylated drugs before. The prevalence of anti-PEG antibodies in ALLcohort 1 was within the range of reported prevalences $(0.2$ to $72 \%) .14,18,19,22,26-30$ However, it must be acknowledged that it is difficult to compare the prevalence between different studies when different methods and cut-points were used (Online Supplementary Tables S1 and S2).

The reported effects of pre-existing anti-PEG antibodies on the efficacy and tolerability of pegylated drugs vary. ${ }^{14,17-}$ 19,31,32 Unexpectedly, the first administration of PEG-ASNase did not trigger the formation of further anti-PEG antibodies. Instead, anti-PEG antibody levels and their prevalence decreased, which was different from a typical hypersensitivity reaction to PEG-ASNase that developed after repeated administration of PEG-ASNase..$^{23,33-35}$ We also observed no increase in anti-PEG antibodies in the seven patients with hypersensitivity reaction at first exposure to PEGASNase.

Four of these patients had pre-existing anti-PEG IgG (MFI 28) (Table 3). This association was significant and the risk 
Table 2. Distribution of PEG-asparaginase activities below and above various thresholds among patients with and without preexisting anti-PEG antibodies.

\begin{tabular}{|c|c|c|c|c|c|c|}
\hline \multirow{2}{*}{$\begin{array}{l}\text { PEG-ASNase } \\
\text { levels }\end{array}$} & \multicolumn{2}{|c|}{ Pre-existing anti-PEG IgG } & \multirow{2}{*}{\multicolumn{2}{|c|}{ odds ratio $(95 \%$ CI) }} & \multirow[b]{2}{*}{ P-value } & \multirow[b]{2}{*}{ adjusted P-value } \\
\hline & $\begin{array}{c}\text { yes } \\
{[M F \mid \geq 8]}\end{array}$ & $\begin{array}{c}n 0 \\
{[M F \mid<8]}\end{array}$ & & & & \\
\hline $\begin{array}{l}<\text { LLOQ } \\
\geq \text { LLOQ }\end{array}$ & $\begin{array}{c}2 \\
88\end{array}$ & $\begin{array}{c}4 \\
552\end{array}$ & 3.14 & $(0.57-17.4)$ & 0.198 & 0.535 \\
\hline $\begin{array}{l}<100 \mathrm{U} / \mathrm{L} \\
\geq 100 \mathrm{U} / \mathrm{L}\end{array}$ & $\begin{array}{c}6 \\
84\end{array}$ & $\begin{array}{c}7 \\
549\end{array}$ & 5.60 & $(5.6-17.1)$ & 0.005 & 0.085 \\
\hline $\begin{array}{l}<400 \mathrm{U} / \mathrm{L} \\
\geq 400 \mathrm{U} / \mathrm{L}\end{array}$ & $\begin{array}{l}35 \\
55\end{array}$ & $\begin{array}{l}116 \\
440\end{array}$ & 2.41 & $(1.51-3.86)$ & 0.0005 & 0.002 \\
\hline $\begin{array}{l}\text { PEG-ASNase } \\
\text { levels }\end{array}$ & $\begin{array}{c}\text { Pre-existin } \\
\text { yes } \\
{[M F \mid \geq 2]}\end{array}$ & $\begin{array}{c}\text { I-PEG IgM } \\
\text { no } \\
{[M F \mid<2]}\end{array}$ & \multicolumn{2}{|c|}{ odds ratio (95\% CI) } & P-value & adjusted P-value \\
\hline $\begin{array}{l}<\text { LLOQ } \\
\geq \text { LLOQ }\end{array}$ & $\begin{array}{c}2 \\
189\end{array}$ & $\begin{array}{c}4 \\
451\end{array}$ & 1.19 & $(0.22-6.57)$ & 1 & 0.535 \\
\hline $\begin{array}{l}<100 \mathrm{U} / \mathrm{L} \\
\geq 100 \mathrm{U} / \mathrm{L}\end{array}$ & $\begin{array}{c}6 \\
185\end{array}$ & $\begin{array}{c}7 \\
448\end{array}$ & 2.08 & $(0.69-6.62)$ & 0.22 & 0.476 \\
\hline $\begin{array}{l}<400 \mathrm{U} / \mathrm{L} \\
\geq 400 \mathrm{U} / \mathrm{L}\end{array}$ & $\begin{array}{c}55 \\
136 \\
\end{array}$ & $\begin{array}{c}96 \\
359 \\
\end{array}$ & 1.51 & $(1.03-2.22)$ & 0.045 & 0.021 \\
\hline
\end{tabular}

For each patient of ALL-cohort 1 only the lowest PEG-ASNase activities determined within 15 days after administration of 2500 U/m² PEG-ASNase were evaluated. PEG-ASNase: polyethylene glycol asparaginase; MFI: mean fluorescence intensity; $95 \% \mathrm{CI}$ : $95 \%$ confidence interval; LLOQ: lower limit of quantification; >: above threshold; <: below threshold.

for hypersensitivity reaction increased with increasing antiPEG IgG levels prior to PEG-ASNase administration. A significant association between pre-existing anti-PEG antibodies and first-exposure hypersensitivity reaction was also documented in the RADAR phase IIb clinical trial, which evaluated pegnivacogin, a 2'-fluoropyrimidine-modified RNA aptamer, in patients with acute coronary syndrome. ${ }^{31}$ Among the six patients with the highest anti-PEG antibody levels prior to pegnivacogin administration, three suffered from a first-exposure hypersensitivity reaction. The firstexposure hypersensitivity reactions in the RADAR phase IIb clinical trial affected only $0.5 \%$ of patients but were considered serious and led to early termination of the trial. ${ }^{31}$ In ALL-cohort 1 the first-exposure hypersensitivity reactions to PEG-ASNase were, however, only moderate (CTCAE grade 2).

Symptoms of moderate hypersensitivity reactions (CTCAE grade $\leq 2$ ) and infusion-related adverse events are often difficult to distinguish. ${ }^{36}$ Typically, hypersensitivity reactions occur after re-challenge to the antigen and are associated with an increase in antibodies, which can persist in the blood for up to several weeks. ${ }^{23,37}$ Since this was not the case in patients with first-exposure hypersensitivity reactions to PEG-ASNase and only moderate hypersensitivity reactions (CTCAE grade 2) occurred, one might conclude that pre-existing anti-PEG IgG simply predispose to mild hypersensitivity reactions for which re-challenge with PEG-ASNase may be possible. The two ALL patients with pre-existing anti-PEG IgG who developed first-exposure hypersensitivity reactions to PEG-ASNase and did not switch to Erwinia ASNase tolerated their subsequent PEG-ASNase administrations well.

Pre-existing anti-PEG antibodies are most likely triggered by repeated contact with PEG-containing products of daily life, such as cosmetics, pharmaceuticals and food. Depending on the nature of the PEG-containing compound, different immunological mechanisms are supposed to facilitate the anti-PEG antibody response. ${ }^{28,38,39}$ Experiments in nude mice showed that pegylated proteins induced the production of anti-PEG IgM in a T-cell- dependent manner, whereas the induction of anti-PEG IgM by pegylated liposomes was T-cell independent. ${ }^{39,40}$ Furthermore, studies in animals indicated that these different immunological processes may also lead to antibodies with different properties. ${ }^{40}$ Similar processes might also be feasible in humans and might explain why patients with pre-existing antibodies showed different antibody responses after their first PEG-ASNase dose than patients who developed a hypersensitivity reaction to the PEG-ASNase after repeated administrations and in whom the PEG covalently bound to the bacterial ASNase acted as a hapten. ${ }^{23,37}$ According to the "Consensus expert recommendations for identification and management of ASNase hypersensitivity and silent inactivation" discontinuation of treatment is recommended for grade $\geq 2$ allergic reactions. ${ }^{24}$ Recently, the National Comprehensive Cancer Network clinical practice guidelines and other expert reviews on ASNase hypersensitivity recommended switching of ASNase preparations only in the event of grade $\geq 3$, severe or life-threatening allergic or anaphylactic reactions. ${ }^{36,41-43}$ In addition, because of the repeated shortage of Erwinia ASNase, various strategies were evaluated in order to avoid or delay a switch to Erwinia ASNase as long as possible. ${ }^{36,44,45}$ Thus, PEG-ASNase was either generally administered under premedication or in the case of hypersensitivity reactions grade $\leq 2$ under premedication at initially reduced infusion rates. In each case, PEG-ASNase activity was monitored to detect silent inactivation or premedication-masked hypersensitivity reactions.

Pre-existing anti-PEG antibodies reduced the PEGASNase activity levels as a function of concentration, but did not reduce the PEG-ASNase activity levels to such an extent that the criteria of silent inactivation were fulfilled. Silent inactivation (or subclinical hypersensitivity reaction) is characterized by the development of antibodies without overt symptoms of a hypersensitivity reaction..$^{24,25}$ According to the "Consensus expert recommendations for identification and management of asparaginase hypersensitivity and silent inactivation" silent inacti- 
Table 3. Distribution of pre-existing anti-PEG IgG levels (at various thresholds) among patients of ALL-cohort 1 with and without first-exposure hypersensitivity reactions to PEG-asparaginase.

\begin{tabular}{|c|c|c|c|c|c|c|c|c|c|}
\hline \multirow{2}{*}{$\begin{array}{l}\text { Anti-PEG IgG level } \\
\text { prior to first } \\
\text { PEG-ASNase }\end{array}$} & \multicolumn{2}{|c|}{$\begin{array}{l}\text { Hypersensitivity reaction } \\
\text { to first PEG-ASNase }\end{array}$} & \multicolumn{7}{|c|}{ Statistics } \\
\hline & No & Yes & $P^{a}$ & $\begin{array}{l}\text { Odds ratio } \\
(95 \% \text { CI) }\end{array}$ & $\begin{array}{l}\text { Relative risk } \\
\text { (95\% Cl) }\end{array}$ & $\begin{array}{c}\text { PPV } \\
\text { (95\% Cl) }\end{array}$ & $\begin{array}{c}\text { NPV } \\
(95 \% \text { CI) }\end{array}$ & $\begin{array}{l}\text { Sensitivity } \\
(95 \% \text { CI) }\end{array}$ & $\begin{array}{l}\text { Specificity } \\
\text { ( } 95 \% \text { CI) }\end{array}$ \\
\hline $\begin{array}{l}\mathrm{MFI}>25 \\
\mathrm{MFI} \leq 25\end{array}$ & $\begin{array}{c}7 \\
659\end{array}$ & $\begin{array}{l}3 \\
4\end{array}$ & 0.00008 & $\begin{array}{c}70.6 \\
(10.1-497)\end{array}$ & $\begin{array}{c}49.7 \\
(9.33-228)\end{array}$ & $\begin{array}{c}0.30 \\
(0.07-0.65)\end{array}$ & $\begin{array}{c}0.99 \\
(0.98-1.00)\end{array}$ & $\begin{array}{c}0.43 \\
(0.10-0.82)\end{array}$ & $\begin{array}{c}0.99 \\
(0.98-1.00)\end{array}$ \\
\hline $\begin{array}{l}\mathrm{MFI}>15 \\
\mathrm{MFI} \leq 15\end{array}$ & $\begin{array}{l}26 \\
640\end{array}$ & $\begin{array}{l}4 \\
3\end{array}$ & 0.0001 & $\begin{array}{c}32.8 \\
(5.80-197)\end{array}$ & $\begin{array}{c}28.6 \\
(5.57-157)\end{array}$ & $\begin{array}{c}0.13 \\
(0.04-0.31)\end{array}$ & $\begin{array}{c}0.99 \\
(0.99-1.00)\end{array}$ & $\begin{array}{c}0.57 \\
(0.18-0.90)\end{array}$ & $\begin{array}{c}0.96 \\
(0.94-0.97)\end{array}$ \\
\hline $\begin{array}{l}\mathrm{MFI}>8 \\
\mathrm{MFI} \leq 8\end{array}$ & $\begin{array}{c}90 \\
576\end{array}$ & $\begin{array}{l}4 \\
3\end{array}$ & 0.009 & $\begin{array}{c}8.53 \\
(1.59-48.9)\end{array}$ & $\begin{array}{c}8.21 \\
(1.58-45.7)\end{array}$ & $\begin{array}{c}0.04 \\
(0.01-0.11)\end{array}$ & $\begin{array}{c}0.99 \\
(0.98-1.00)\end{array}$ & $\begin{array}{c}0.57 \\
(0.18-0.90)\end{array}$ & $\begin{array}{c}0.86 \\
(0.84-0.89) \\
\end{array}$ \\
\hline
\end{tabular}

${ }^{\mathrm{a}}$ Fisher exact test; PEG-ASNase: polyethylene glycol asparaginase; $95 \% \mathrm{CI}$ : 95\% confidence interval; PPV: positive predictive value; NPV: negative predictive value; MFI: mean fluorescence intensity

vation of PEG-ASNase is defined by PEG-ASNase activities $\leq 100 \mathrm{U} / \mathrm{L}$ within 7 days and undetectable PEGASNase activities within 14 days after administration. ${ }^{24}$ Neutralizing antibodies but also an accelerated elimination of antigen-antibody complexes are being discussed as the underlying cause for the rapid decrease in ASNase activity. ${ }^{24,25}$ We could not detect any inhibition of asparagine hydrolysis by anti-PEG antibodies (Online Supplementary Figure S4). Animal studies have shown an increased clearance of pegylated proteins, liposomes and nanoparticles in the presence of anti-PEG IgM and antiPEG IgG. ${ }^{15,16}$ In nude mice, anti-PEG IgM induced a rapid clearance of pegylated protein from serum with simultaneous accumulation in the liver. Similar processes could also be conceivable in humans. The increased clearance of PEG uricase in gout patients was associated with an increase in anti-PEG IgG and IgM levels. ${ }^{18,19}$ The lower PEG-ASNase activity levels in patients with pre-existing antibodies might have been caused by an increased clearance of PEG-ASNase.

When comparing the distribution of PEG-ASNase activities above and below $400 \mathrm{U} / \mathrm{L}, 100 \mathrm{U} / \mathrm{L}$ and the LLOQ (5 $\mathrm{U} / \mathrm{L})$, we found a significant difference between patients with and without pre-existing anti-PEG antibodies only at $400 \mathrm{U} / \mathrm{L}$. The $400 \mathrm{U} / \mathrm{L}$ value was chosen in addition to the generally accepted target activity of $100 \mathrm{U} / \mathrm{L}$ and the LLOQ because $400 \mathrm{U} / \mathrm{L}$ have been shown to result in deeper asparagine depletion. ${ }^{46,47}$ This higher activity level and its associated glutaminase activity have been suggested to increase the effectiveness of ASNase against leukemic blasts with residual asparagine synthetase activity. ${ }^{48}$ Several studies have recently shown that PEGASNase clearance in a patient can vary significantly between different parts of the protocol. ${ }^{49,50}$ Thus, the effect of increased PEG-ASNase clearance due to preexisting antibodies on the intensity of ASNase therapy would depend on the dose and concomitant ALL treatment. Therefore, the effects of pre-existing anti-PEG antibodies on the pharmacokinetics of PEG-ASNase must be determined separately for each protocol.

In summary, we observed a considerable number of patients with pre-existing antibodies against PEG. AntiPEG antibody kinetics after PEG-ASNase administration were not the same in patients with pre-existing antibodies as in patients with hypersensitivity reactions after repeated PEG-ASNase administration. ${ }^{23,37}$ Pre-existing anti-PEG antibodies may cause mild to moderate symptoms of hypersensitivity reaction with the first administration of PEG-ASNase, which might be addressed by rechallenge. They do not inhibit PEG-ASNase activity but lower PEG-ASNase activity levels, which, depending on the dose and protocol, may interfere with the targeted PEG-ASNase treatment intensity.

\section{Disclosures}

MS and the ALL-BFM Study Center have received funding from medac, Shire, SigmaTau, Servier, and Jazz Pharmaceuticals for research, and MS has participated in advisory boards. CR received institutional grants for PEG-asparaginase pharmacological studies aimed at the therapeutic monitoring of asparaginase activity within the AIEOP-BFM ALL 2009 study, as well as fees for participation in advisory boards and invited lectures for the companies involved in marketing different asparaginase products, medac GmbH, Sigma-Tau, Baxalta, Shire, Servier. CLK has held invited talks for Sigma tau, Erytech and Jazz Pharmaceuticals, received honoraria for consultancy from Erytech and travel expenses from Erytech and Servier. AA has consulting and advisory roles for Jazz Pharmaceuticals, and has also received travel and accommodation fees and expenses from Jazz Pharmaceuticals. AM has received honoraria from Baxter. $J B$ has served personally as a consultant and participated in advisory as well as in safety boards for medac GmbH; he has received support for travel from Eusa Pharma, Jazz Pharmaceuticals, Baxalta Shire and Servier; has held invited lectures for medac GmbH, Eusa Pharma, Jazz Pharmaceuticals, Baxalta, Servier, Shire and Sigma-Tau; and, in addition, he has received institutional grants in the context of ASNase drug monitoring from more or less all ASNase providers contributing to the therapeutic drug monitoring program, including medac GmbH, Eusa Pharma, Jazz Pharmaceuticals, Baxalta, Servier, Shire, and Sigma-Tau (all representing the varying marketing authorization holders of $E$. coli ASNase, PEG-ASNase and Erwinase). NG has received research support from Jazz Pharmaceuticals and honoraria as an advisory board member from Jazz Pharmaceuticals and Baxalta. The other authors declare that they have no potential conflicts of interest.

\section{Contributions}

$A K, C L K, G W$ and $J B$ designed the research. $C R, M Z u, V C$, $C N, A A, A M, M S, J B, A v S$ and NG collected data. AA, CN, $M Z u, M F, C L K, G H$ and JB analyzed and interpreted the data. $A K, G W, J G, M Z i, A M$ and $C L K$ performed the statistical analysis. $A K$ and $C L K$ wrote the manuscript.

\section{Acknowledgments}

The authors would like to thank Ms Schulze Westhoff, Ms Hoogestraat and Ms Günter for their excellent technical assistance. We acknowledge support from the Open Access Publication Fund of the University of Muenster. 


\section{Funding}

This work was supported by the Deutsche José Carreras Leukämie-Stiftung e.V. (DJCLSR 13/01). The monitoring of PEG-ASNase activities and of antibodies against PEG-ASNase and native E. coli ASNase was supported by Servier (Suresnes, France), Shire (Lexington, USA), Baxalta (Westlake Village, USA), Sigma Tau Pharmaceuticals (Zofingen, Switzerland) and medac GmbH (Wedel, Germany). In Australia, ASNase monitoring and the collection of samples for antibody determination for the
AIEOP-BFM ALL 2009 trial were funded by the Kids Cancer Alliance (KCA), a Translational Cancer Research Center of the Cancer Institute of New South Wales (15/TRC/1-04). CN is supported by the Lenkemia Research and Support Fund at The Children's Hospital at Westmead (Australia). In Austria monitoring of ASNase activity and collection of samples for antibody determination for the AIEOP-BFM ALL 2009 trial were funded by the St. Anna Kinderkrebsforschung (BFM-Austria). The work is part of the thesis of $A K$.

\section{References}

1. Fruijtier-Pölloth C. Safety assessment on polyethylene glycols (PEGs) and their derivatives as used in cosmetic products. Toxicology. 2005;214(1-2):1-38.

2. Caliceti P, Veronese FM. Pharmacokinetic and biodistribution properties of poly(ethylene glycol)-protein conjugates. Adv Drug Deliv Rev. 2003;55(10):1261-1277.

3. Harris JM, Chess RB. Effect of pegylation on pharmaceuticals. Nat Rev Drug Discov. 2003;2(3):214-221.

4. Werle M, Bernkop-Schnürch A. Strategies to improve plasma half life time of peptide and protein drugs. Amino Acids. 2006;30(4):351367.

5. Sherman MR, Saifer MGP, Perez-Ruiz F. $\mathrm{PEG}$-uricase in the management of treatment-resistant gout and hyperuricemia. Adv Drug Deliv Rev. 2008;60(1):59-68.

6. Dinndorf PA, Gootenberg J, Cohen MH, Keegan P, Pazdur R. FDA drug approval summary: pegaspargase (oncaspar) for the first-line treatment of children with acute lymphoblastic leukemia (ALL). Oncologist. 2007;12(8):991-998.

7. Barnard DL. Pegasys (Hoffmann-La Roche). Curr Opin Investig Drugs. 2001;2(11):15301538.

8. Graham ML. Pegaspargase: a review of clinical studies. Adv Drug Deliv Rev. 2003;55(10):1293-1302.

9. Pieters R, Hunger SP, Boos J, et al. L-asparaginase treatment in acute lymphoblastic leukemia: a focus on Erwinia asparaginase. Cancer. 2011;117(2):238-249.

10. Hunger SP, Mullighan CG. Acute lymphoblastic leukemia in children. $\mathrm{N}$ Engl J Med. 2015;373(16):1541-1552.

11. Place AE, Stevenson KE, Vrooman LM, et al. Intravenous pegylated asparaginase versus intramuscular native Escherichia coli Lasparaginase in newly diagnosed childhood acute lymphoblastic leukaemia (DFCI 05001): a randomised, open-label phase 3 trial. Lancet Oncol. 2015;16(16):1677-1690.

12. Zeidan A, Wang ES, Wetzler M. Pegasparaginase: where do we stand? Expert Opin Biol Ther. 2009;9(1):111-119.

13. Rytting $M$. Peg-asparaginase for acute lymphoblastic leukemia. Expert Opin Biol Ther. 2010;10(5):833-839.

14. Yang Q, Lai SK. Anti-PEG immunity: emergence, characteristics, and unaddressed questions. Wiley Interdiscip Rev Nanomed Nanobiotechnol. 2015;7(5):655-677

15. Poppenborg SM, Wittmann J, Walther W, et al. Impact of anti-PEG IgM antibodies on the pharmacokinetics of pegylated asparaginase preparations in mice. Eur J Pharm Sci. 2016;91:122-130

16. Ishida T, Kashima S, Kiwada H. The contribution of phagocytic activity of liver macrophages to the accelerated blood clearance $(\mathrm{ABC})$ phenomenon of PEGylated lipo- somes in rats. J Control Release. 2008;126 (2):162-165

17. Myler H, Hruska MW, Srinivasan S, et al. Anti-PEG antibody bioanalysis: a clinical case study with PEG-IFN- $\gamma$ - $1 \mathrm{a}$ and PEG-IFN$\alpha 2 \mathrm{a}$ in naive patients. Bioanalysis. 2015;7(9):1093-1106.

18. Ganson NJ, Kelly SJ, Scarlett E, Sundy JS, Hershfield MS. Control of hyperuricemia in subjects with refractory gout, and induction of antibody against poly(ethylene glycol) (PEG), in a phase I trial of subcutaneous PEGylated urate oxidase. Arthritis Res Ther. 2006;8(1):R12.

19. Hershfield MS, Ganson NJ, Kelly SJ, Scarlett EL, Jaggers DA, Sundy JS. Induced and preexisting anti-polyethylene glycol antibody in a trial of every 3 -week dosing of pegloticase for refractory gout, including in organ transplant recipients. Arthritis Res Ther. 2014;16 (2):R63.

20. U.S. Department of Health and Human Services, Food and Drug Administration. Guidance for Industry: Immunogenicity Assessment for Therapeutic Protein Products. https://www.fda.gov/regulatoryinformation/search-fda-guidance-documents/immunogenicity-assessment-therapeutic-protein-products. Accessed 11.11.2020

21. Parenky A, Myler H, Amaravadi L, et al. New FDA draft guidance on immunogenicity. AAPS J. 2014;16(3):499-503

22. Armstrong JK, Hempel G, Koling S, et al. Antibody against poly(ethylene glycol) adversely affects PEG-asparaginase therapy in acute lymphoblastic leukemia patients. Cancer. 2007;110(1):103-111.

23. Liu Y, Smith CA, Panetta JC, et al. Antibodies predict pegaspargase allergic reactions and failure of rechallenge. J Clin Oncol. 2019;37(23):2051-2061.

24. van der Sluis IM, Vrooman LM, Pieters R, et al. Consensus expert recommendations for identification and management of asparaginase hypersensitivity and silent inactivation. Haematologica. 2016;101(3):279-285.

25. Asselin B, Rizzari C. Asparaginase pharmacokinetics and implications of therapeutic drug monitoring. Leuk Lymphoma. 2015;56 (8):2273-2280.

26. Richter AW, Akerblom E. Polyethylene glycol reactive antibodies in man: titer distribution in allergic patients treated with monomethoxy polyethylene glycol modified allergens or placebo, and in healthy blood donors. Int Arch Allergy Appl Immunol. 1984;74(1):36-39.

27. Liu Y, Reidler H, Pan J, et al. A double antigen bridging immunogenicity ELISA for the detection of antibodies to polyethylene glycol polymers. J Pharmacol Toxicol Methods. 2011;64(3):238-245

28. Lubich C, Allacher P, de la Rosa M, et al. The mystery of antibodies against polyethylene glycol (PEG) - what do we know? Pharm
Res. 2016;33(9):2239-2249.

29. Chen B-M, Su Y-C, Chang C-J, et al. Measurement of pre-existing IgG and IgM antibodies against polyethylene glycol in healthy individuals. Anal Chem. 2016;88 (21):10661-10666

30. Yang Q, Jacobs TM, McCallen JD, et al. Analysis of pre-existing $\operatorname{IgG}$ and $\operatorname{IgM}$ antibodies against polyethylene glycol (PEG) in the general population. Anal Chem. 2016;88(23):11804-11812

31. Ganson NJ, Povsic TJ, Sullenger BA, et al. Pre-existing anti-polyethylene glycol antibody linked to first-exposure allergic reactions to pegnivacogin, a PEGylated RNA aptamer. J Allergy Clin Immunol. 2016;137(5):1610-1613

32. Tillmann H, Ganson NJ, Patel K, et al. High prevalence of pre-existing antibodies against polyethylene glycol (PEG) in hepatitis C (HCV) patients which is not associated with impaired response to PEG-interferon. J Hepatol. 2010;52:S129.

33. Henriksen LT, Nersting J, Raja RA, et al. Cerebrospinal fluid asparagine depletion during pegylated asparaginase therapy in children with acute lymphoblastic leukaemia. Br J Haematol. 2014;166(2):213220.

34. Henriksen LT, Harila-Saari A, Ruud E, et al. PEG-asparaginase allergy in children with acute lymphoblastic leukemia in the NOPHO ALL2008 protocol. Pediatr Blood Cancer. 2015;62(3):427-433.

35. Tong WH, Pieters R, Kaspers GJL, et al. A prospective study on drug monitoring of PEGasparaginase and Erwinia asparaginase and asparaginase antibodies in pediatric acute lymphoblastic leukemia. Blood. 2014;123(13):2026-2033.

36. Burke MJ, Rheingold SR. Differentiating hypersensitivity versus infusion-related reactions in pediatric patients receiving intravenous asparaginase therapy for acute lymphoblastic leukemia. Leuk Lymphoma. 2017;58(3):540-551.

37. Willer A, Gerss J, König T, et al. AntiEscherichia coli asparaginase antibody levels determine the activity of second-line treatment with pegylated $\mathrm{E}$ coli asparaginase: a retrospective analysis within the ALL-BFM trials. Blood. 2011;118(22):5774-5782.

38. Mond JJ, Vos Q, Lees A, Snapper CM. T cell independent antigens. Curr Opin Immunol. 1995;7(3):349-354.

39. Ishida T, Wang $X$, Shimizu T, Nawata K, Kiwada H. PEGylated liposomes elicit an anti-PEG IgM response in a $\mathrm{T}$ cell-independent manner. J Control Release. 2007;122(3): 349-355.

40. Mima Y, Hashimoto Y, Shimizu T, Kiwada $\mathrm{H}$, Ishida $\mathrm{T}$. Anti-PEG IgM is a major contributor to the accelerated blood clearance of polyethylene glycol-conjugated protein. Mol Pharm. 2015;12(7):2429-2435

41. Marini BL, Perissinotti AJ, Bixby DL, Brown 
J, Burke PW. Catalyzing improvements in ALL therapy with asparaginase. Blood Rev. 2017;31(5):328-338.

42. Benitez L, Perissinotti AJ, Santarossa M, Marini BL. Pharmacokinetic and clinical considerations for monitoring asparaginase activity levels during pegaspargase therapy. Pediatr Blood Cancer. 2015;62(6):1115.

43. Verma A, Chen K, Bender C, Gorney N, Leonard W, Barnette P. PEGylated E. coli asparaginase desensitization: an effective and feasible option for pediatric patients with acute lymphoblastic leukemia who have developed hypersensitivity to pegaspargase in the absence of asparaginase Erwinia chrysanthemi availability. Pediatr Hematol Oncol. 2019;36(5):277-286.

44. Marini BL, Brown J, Benitez L, et al. A singlecenter multidisciplinary approach to managing the global Erwinia asparaginase short- age. Leuk Lymphoma. 2019;60(12):2854 2868.

45. Cooper SL, Young DJ, Bowen CJ, Arwood NM, Poggi SG, Brown PA. Universal premedication and therapeutic drug monitoring for asparaginase-based therapy prevents infusion-associated acute adverse events and drug substitutions. Pediatr Blood Cancer. 2019;66(8):e27797.

46. Angiolillo AL, Schore RJ, Devidas M, et al. Pharmacokinetic and pharmacodynamic properties of calaspargase pegol Escherichia coli L-asparaginase in the treatment of patients with acute lymphoblastic leukemia: results from Children's Oncology Group Study AALL07P4. J Clin Oncol. 2014;32(34): 3874-3882.

47. Avramis VI, Panosyan EH. Pharmacokinetic/ pharmacodynamic relationships of asparaginase formulations: the past, the present and recommendations for the future. Clin Pharmacokinet. 2005;44 (4):367-393.

48. Chan WK, Lorenzi PL, Anishkin A, et al. The glutaminase activity of L-asparaginase is not required for anticancer activity against ASNS-negative cells. Blood. 2014;123(23): 3596-3606.

49. Kloos ROH, Mathôt R, Pieters R, van der Sluis IM. Individualized dosing guidelines for PEGasparaginase and factors influencing the clearance: a population pharmacokinetic model. Haematologica. 2021;106(5):1254 1261.

50. Lanvers-Kaminsky C, Niemann A, Eveslage $\mathrm{M}$, et al. Asparaginase activities during intensified treatment with pegylated E. coli asparaginase in adults with newly-diagnosed acute lymphoblastic leukemia. Leuk Lymphoma. 2020;61(1):138-145. 